\title{
DETERMINING A PARTICULAR SOLUTION FOR SYSTEMS OF LINEAR DIFFERENTIAL EQUATIONS WITH CONSTANT COEFFICIENTS THAT HAVE ON THE COLUMN OF FREE TERMS FUNCTIONS SUCH AS
}$$
e^{a \cdot x} \cdot P(x) \cdot \cos ^{n} x \text { or } e^{b \cdot x} \cdot Q(x) \cdot \sin ^{n} x
$$ \\ Vasile CĂRUŢAŞU, Ioan VIRCA \\ "Nicolae Bălcescu" Land Forces Academy, Sibiu, Romania \\ v.carutasu@yahoo.com, virca_ioan@yahoo.com
}

\begin{abstract}
The determination of a particular solution for the systems of linear differential equations with constant coefficients that have on the column of free terms functions such as $e^{a \cdot x} \cdot P(x) \cdot \cos ^{n} x$ orland $e^{b \cdot x} \cdot Q(x) \cdot \sin ^{n} x, n \in N$, is based on the expansion of $\cos ^{n} x$ and $\sin ^{n} x$, and, on the other hand, on how a particular solution for a column of free terms with functions such as $e^{a \cdot x} \cdot P(x) \cdot \cos ^{n} x$ or $e^{b \cdot x} \cdot Q(x) \cdot \sin ^{n} x, n \in N$ looks like. We can also write the way a particular solution looks like when we have a combination of two or more functions on the form $e^{a \cdot x} \cdot P(x) \cdot \cos ^{n} x$ or $e^{b \cdot x} \cdot Q(x) \cdot \sin ^{n} x, n \in N$ in the column of free terms.
\end{abstract}

Keywords: systems of linear differential equations with constant coefficients, particular solution, general solution, column of free terms with functions such as $P(x) \cdot e^{a \cdot x} \cdot \cos ^{n} x$ or/and $Q(x) \cdot e^{b \cdot x} \cdot \sin ^{n} x$

\section{Introduction}

Based on the expansion of $\cos ^{n} x$ and $\sin ^{n} x$, in this study it is presented the way in which a particular solution for the systems of linear differential equations with constant coefficients that has on the column $e^{a \cdot x} \cdot P(x) \cdot \cos ^{n} x$ or/and $e^{b \cdot x} \cdot Q(x) \cdot \sin ^{n} x, n \in N$ can be directly constructed similarly as it was done in [1]. As we mentioned before, we will start from the expansion of $\cos ^{n} x$ or $\sin ^{n} x$, according to the relations below:

of free terms functions such as

$$
\begin{aligned}
& \cos ^{n} x=\frac{1}{2^{n-1}} \cdot\left[\sum_{\substack{r=0 \\
2 \cdot r<n}} C_{n}^{r} \cdot \cos (n-2 \cdot r) \cdot x\right]+\frac{1}{2^{n}} \cdot C_{n}^{n / 2}, n \text { even; } \\
& \cos ^{n} x=\frac{1}{2^{n-1}} \cdot\left[\sum_{\substack{r=0 \\
2 \cdot r<n}} C_{n}^{r} \cdot \cos (n-2 \cdot r) \cdot x\right], n \text { odd }
\end{aligned}
$$




$$
\begin{aligned}
& \sin ^{n} x=\frac{(-1)^{n / 2}}{2^{n-1}} \cdot\left[\sum_{\substack{r=0 \\
2 \cdot r<n}}(-1)^{r} \cdot C_{n}^{r} \cdot \cos (n-2 \cdot r) \cdot x\right]+\frac{1}{2^{n}} \cdot C_{n}^{n / 2, n \text { even }} \\
& \sin ^{n} x=\frac{(-1)^{(n-1) / 2}}{2^{n-1}} \cdot\left[\sum_{\substack{r=0 \\
2 \cdot r<n}}(-1)^{r} \cdot C_{n}^{r} \cdot \sin (n-2 \cdot r) \cdot x\right], n \text { odd, }
\end{aligned}
$$

relations which, as we have also mentioned in the case of $n$-th order linear differential equations with constant coefficients, are proved using mathematical induction.

\section{Problem description}

Let us consider that we have a system of linear differential equations with constant coefficients, such as

$$
\left\{\begin{array}{l}
y_{1}^{\prime}(x)=a_{11} \cdot y_{1}(x)+a_{12} \cdot y_{2}(x)+\ldots+a_{1 n} \cdot y_{n}(x)+f_{1}(x) \\
y_{2}^{\prime}(x)=a_{21} \cdot y_{1}(x)+a_{22} \cdot y_{2}(x)+\ldots+a_{2 n} \cdot y_{n}(x)+f_{2}(x) \\
\vdots \\
y_{n}^{\prime}(x)=a_{n 1} \cdot y_{1}(x)+a_{n 2} \cdot y_{2}(x)+\ldots+a_{n n} \cdot y_{n}(x)+f_{n}(x)
\end{array}\right.
$$

where $x \in I \subset \boldsymbol{R}$ is an interval of $\boldsymbol{R}$, and $f_{i}, \quad$ The homogeneous system attached to the $i \in \overline{1, n}$, are functions that will be defined system (5) will be like below.

$$
\left\{\begin{array}{l}
y_{1}^{\prime}(x)=a_{11} \cdot y_{1}(x)+a_{12} \cdot y_{2}(x)+\ldots+a_{1 n} \cdot y_{n}(x) \\
y_{2}^{\prime}(x)=a_{21} \cdot y_{1}(x)+a_{22} \cdot y_{2}(x)+\ldots+a_{2 n} \cdot y_{n}(x) \\
\vdots \\
y_{n}^{\prime}(x)=a_{n 1} \cdot y_{1}(x)+a_{n 2} \cdot y_{2}(x)+\ldots+a_{n n} \cdot y_{n}(x)
\end{array}\right.
$$

where $x \in I \subset \boldsymbol{R}$ is an interval of $\boldsymbol{R}$.

We will assume that

$$
\begin{aligned}
f_{m}(x)= & \sum_{i=1}^{p_{m}} P_{m i}(x) \cdot e^{\alpha_{m i} \cdot x} \cdot \cos ^{b_{m i}} x+\sum_{j=1}^{q_{m}} Q_{m j}(x) \cdot e^{\beta_{m j} \cdot x} \cdot \cos ^{c_{m j}} x+ \\
& +\sum_{k=1}^{r_{m}} R_{m k}(x) \cdot e^{\gamma_{m k} \cdot x} \cdot \sin ^{d_{m k}} x+\sum_{l=1}^{s_{m}} S_{m l}(x) \cdot e^{\delta_{m l} \cdot x} \cdot \sin ^{g_{m l}} x
\end{aligned},
$$

where $\quad m \in \overline{1, n} ; \quad p_{m}, \quad q_{m}, \quad r_{m}, \quad s_{m} \in N^{*}$, $(\forall) m \in \overline{1, n} ; \quad \alpha_{m i}, \quad \beta_{m j}, \quad \gamma_{m k}, \quad \delta_{m l} \quad \in \boldsymbol{R}$, $(\forall) m \in \overline{1, n},(\forall) i \in \overline{1, p_{m}}, \quad(\forall) j \in \overline{1, q_{m}}$, $(\forall) k \in \overline{1, r_{m}}$ and $(\forall) l \in \overline{1, s_{m}} ; b_{m i}, c_{m j}, d_{m k}$, $g_{m l} \in N^{*}, b_{m i}, d_{m k}$ even numbers and $c_{m j}, g_{m l}$ odd numbers, $\quad(\forall) m \in \overline{1, n},(\forall) i \in \overline{1, p_{m}}$, $(\forall) j \in \overline{1, q_{m}}, \quad(\forall) k \in \overline{1, r_{m}}$ si $\quad(\forall) l \in \overline{1, s_{m}} ;$ and $P_{m i}, Q_{m j}, R_{m k}, S_{m l}$ are plynomials, $(\forall) m \in \overline{1, n},(\forall) i \in \overline{1, p_{m}}, \quad(\forall) j \in \overline{1, q_{m}}$, $(\forall) k \in \overline{1, r_{m}}$ and $(\forall) l \in \overline{1, s_{m}}$.

As we mentioned before, the particular solution of the system (5), in which the free terms $f_{i}(x), i \in \overline{1, n}$ are functions of the type (7), will be directly constructed similarly as it was done in [1]. 


\section{The form of the particular solution and the exemplification of its determination}

The characteristic equation attached to the homogeneous system (6) is of the following form:

$$
\left|\begin{array}{crrr}
a_{11}-\lambda & a_{12} & \ldots & a_{1 n} \\
a_{21} & a_{22}-\lambda & \ldots & a_{2 n} \\
& \vdots & & \\
a_{n 1} & a_{n 2} & \ldots & a_{n n}-\lambda
\end{array}\right|=0 .
$$

We consider that the equation (8) has the following expansion:

$$
\left|\begin{array}{cccc}
a_{11}-\lambda & a_{12} & \ldots & a_{1 n} \\
a_{21} & a_{22}-\lambda & \ldots & a_{2 n} \\
& \vdots & & \\
a_{n 1} & a_{n 2} & \ldots & a_{n n}-\lambda
\end{array}\right| \begin{aligned}
& (-1)^{n} \cdot\left(\lambda-u_{1}\right)^{n_{1}} \cdot\left(\lambda-u_{2}\right)^{n_{2}} \cdot \ldots \cdot\left(\lambda-u_{h}\right)^{n_{h}} \\
& \cdot\left[\left(\lambda-\left(v_{1}+w_{1} \cdot i\right)\right) \cdot\left(\lambda-\left(v_{1}-w_{1} \cdot i\right)\right)\right]^{m_{1}} \cdot \\
& \cdot\left[\left(\lambda-\left(v_{2}+w_{2} \cdot i\right)\right) \cdot\left(\lambda-\left(v_{2}-w_{2} \cdot i\right)\right)\right]^{m_{2}} \cdot \ldots \cdot \\
& \cdot\left[\left(\lambda-\left(v_{t}+w_{t} \cdot i\right)\right) \cdot\left(\lambda-\left(v_{t}-w_{t} \cdot i\right)\right)\right]^{m_{p}}
\end{aligned}
$$

where $n_{1}+n_{2}+\ldots+n_{q}+2 \cdot m_{1}+2 \cdot m_{2}+\ldots+2 \cdot m_{p}=n$.

If in the expression of the function $f_{m}(x)$ we use the relations (1)-(4), then $f_{m}(x)$ will be like

$$
\begin{gathered}
\sum_{i=1}^{p_{m}} P_{m i}(x) \cdot e^{\alpha_{m i} \cdot x}+\sum_{j=1}^{q_{m}} Q_{m j}(x) \cdot e^{\beta_{m j} \cdot x} \cdot \cos (2 \cdot j \cdot x)+ \\
+\sum_{k=1}^{r_{m}} R_{m k}(x) \cdot e^{\gamma_{m k} \cdot x} \cdot \cos [(2 \cdot k+1) \cdot x]+\sum_{l=1}^{s_{m}} S_{m l}(x) \cdot e^{\delta_{m l} \cdot x} \cdot \sin [(2 \cdot l+1) \cdot x]
\end{gathered}
$$

where, even if we used the same notations as in (7), they no longer have the meaning of that expression. Also, the powers $\beta_{m j},(\forall) j \in \overline{1, q_{m}}$, are not necessarily distinct two by two and the same happens with the powers $\gamma_{m k},(\forall) k \in \overline{1, r_{m}}$ si $\delta_{m l}, \quad(\forall) l \in \overline{1, s_{m}}$. Instead, we can consider the powers $\alpha_{m i}$ as distinct two by two, because if we happen to have the same power we only add the polynomials with which they are multiplied $e^{\alpha_{m i} \cdot x}$.

In order to be able to write a particular solution of the system we will add all the functions that appear in the column of free terms and we will obtain a function like the following

$$
\begin{aligned}
f(x)=\sum_{m=1}^{n} f_{m}(x)= & \sum_{i=1}^{p} P_{i}(x) \cdot e^{\alpha_{i} \cdot x}+\sum_{j=1}^{q} Q_{j}(x) \cdot e^{\beta_{j} \cdot x} \cdot \cos (2 \cdot j \cdot x)+ \\
& +\sum_{k=1}^{r} R_{k}(x) \cdot e^{\gamma_{k} \cdot x} \cdot \cos [(2 \cdot k+1) \cdot x]+\sum_{l=1}^{s} S_{l}(x) \cdot e^{\delta_{l} \cdot x} \cdot \sin [(2 \cdot l+1) \cdot x]
\end{aligned}
$$

where, as previously stated, we can consider that the powers $\alpha_{i}$ are distinct two by two, because if we happen to have the same power $\alpha_{i}=\alpha_{m i}$ for several functions $f_{m}$ we add only the polynomials with which they are multiplied $e^{\alpha_{i} \cdot x}$. In contrast, the powers $\beta_{j},(\forall) j \in \overline{1, q}$, are not necessarily distinct two by two, and the same happens with the powers $\gamma_{k},(\forall) k \in \overline{1, r}$ and $\delta_{l}$, 
$(\forall) l \in \overline{1, s}$. Moreover, we can consider, without restricting the generality, that we do not have terms such as $R_{h}(x)^{\cdot} e^{h \cdot x} \cdot \cos [(2 \cdot h+1) \cdot x] \quad$ and $S_{h}(x)^{\cdot} e^{h \cdot x} \cdot \sin [(2 \cdot h+1) \cdot x]$ in the last two terms of the expression of $f(x)$, because the corresponding term generated in the particular solution, regardless of whether both terms appear or only one of them, is of the form $R_{h}{ }^{\prime}(x)^{\cdot} e^{h \cdot x} \cdot \cos [(2 \cdot h+1) \cdot x]+\quad+$ $S_{h}{ }^{\prime}(x)^{\cdot} e^{h \cdot x} \cdot \sin [(2 \cdot h+1) \cdot x]$, only if both terms appear we take polynomials $R_{h}{ }^{\prime}$ si $S_{h}{ }^{\prime}$, with degree $R_{h}{ }^{\prime}=$ degree $S_{h}{ }^{\prime}=\max$ \{degree $R_{h}$, degree $\left.S_{h}\right\}$.

We also assume that some of the solutions of the characteristic equation (9) are in the following situations:

$$
\begin{array}{lrl}
u_{i_{1}}, u_{i_{2}}, \ldots, \alpha_{i_{a}} \in\left\{\alpha_{i} / i \in \overline{1, p}\right\}, v_{j_{1}}+w_{j_{1}} \cdot i, v_{j_{2}}+w_{j_{2}} \cdot i, \ldots, & \\
v_{j_{b}}+w_{j_{b}} \cdot i \in\left\{\beta_{j}+2 \cdot j \cdot i / j \in \overline{1, q}\right\}, & v_{k_{1}}+w_{k_{1}} \cdot i, & v_{k_{2}}+w_{k_{2}} \cdot i, \\
v_{k_{c}}+w_{k_{c}} \cdot i \in\left\{\gamma_{k}+(2 \cdot k+1) \cdot i / k \in \overline{1, r}\right\}, & v_{l_{1}}+w_{l_{1}} \cdot i, & v_{l_{2}}+w_{l_{2}} \cdot i, \\
v_{l_{d}}+w_{l_{d}} \cdot i \in\left\{\delta_{l}+(2 \cdot l+1) \cdot i / l \in \overline{1, s}\right\} . &
\end{array}
$$

Under these conditions, as in [1], a particular solution of the system (3), in which $f_{m}(x)$, $m \in \overline{1, n}$, are as (7) will be like

$$
\begin{aligned}
& \sum_{i \in\left\{i_{1}, i_{2}, \ldots, i_{a}\right\}} P_{i}^{\prime}(x) \cdot x^{n_{i}} \cdot e^{u_{i} \cdot x}+\sum_{i \in \overline{1, p} \backslash\left\{i_{1}, i_{2}, \ldots, i_{a}\right\}} P_{i}^{\prime}(x) \cdot e^{\alpha_{i} \cdot x}+ \\
& +\sum_{j \in\left\{j_{1}, j_{2}, \ldots, j_{b}\right\}} x^{m_{j}} \cdot e^{v_{j} \cdot x} \cdot\left[Q_{j}^{\prime}(x) \cdot \cos (2 \cdot j \cdot x)+Q_{j}^{\prime \prime}(x) \cdot \sin (2 \cdot j \cdot x)\right]+ \\
& +\sum_{j \in \overline{1, q} \backslash\left\{j_{1}, j_{2}, \ldots, j_{b}\right\}} e^{\beta_{j} \cdot x} \cdot\left[Q_{j}^{\prime}(x) \cdot \cos (2 \cdot j \cdot x)+Q_{j}^{\prime \prime}(x) \cdot \sin (2 \cdot j \cdot x)\right]+ \\
y_{m p}(x)= & +\sum_{k \in\left\{k_{1}, k_{2}, \ldots, k_{c}\right\}} x^{m_{k}} \cdot e^{v_{k} \cdot x} \cdot\left\{R_{k}^{\prime}(x) \cdot \cos [(2 \cdot k+1) \cdot x]+R_{k}^{\prime \prime}(x) \cdot \sin [(2 \cdot k+1) \cdot x]\right\}+, m \in \overline{1, n} \\
& +\sum_{k \in \overline{1, r} \backslash\left\{k_{1}, k_{2}, \ldots, k_{c}\right\}} e^{\gamma_{k} \cdot x} \cdot\left\{R_{k}^{\prime}(x) \cdot \cos [(2 \cdot k+1) \cdot x]+R_{k}^{\prime \prime}(x) \cdot \sin [(2 \cdot k+1) \cdot x]\right\}+ \\
& +\sum_{l \in\left\{l_{1}, l_{2}, \ldots, l_{d}\right\}} x^{m_{l}} \cdot e^{v_{l} \cdot x} \cdot\left\{S_{l}^{\prime}(x) \cdot \cos [(2 \cdot l+1) \cdot x]+S_{l}^{\prime \prime}(x) \cdot \sin [(2 \cdot l+1) \cdot x]\right\}+ \\
& +\sum_{l \in \overline{1, s} \backslash\left\{\left\{1_{1}, l_{2}, \ldots, l_{d}\right\}\right.} e^{\delta_{l} \cdot x} \cdot\left\{S_{l}^{\prime}(x) \cdot \cos [(2 \cdot l+1) \cdot x]+S_{l}^{\prime \prime}(x) \cdot \sin [(2 \cdot l+1) \cdot x]\right\}
\end{aligned}
$$

where degree $P_{i}^{\prime}=$ degree $P_{i}, \quad(\forall) i \in \overline{1, p}$, degree $Q_{j}^{\prime}=$ degree $Q_{j}^{\prime \prime}=$ degree $Q_{j}$, $(\forall) j \in \overline{1, q}$, degree $R_{k}{ }^{\prime}=$ degree $R_{k}{ }^{\prime \prime}=$ degree $R_{k}, \quad(\forall) k \in \overline{1, r}$, degree $S_{l}^{\prime}=$ degree $S_{l}^{\prime \prime}=$ degree $S_{l}, \quad(\forall) l \in \overline{1, s}$.

The construction of the solution (12), takes into account the form of the functions $f_{m}(x)$, $m \in \overline{1, n}$ and the form of the characteristic equation (9).

We will further exemplify the way in which the method described above must be applied. Let us consider the system of linear differential equations with constant coefficients

$$
\left\{\begin{array}{l}
y_{1}^{\prime}(x)=\quad-y_{2}(x)+17 \cdot y_{3}(x)+2 \cdot e^{x} \cdot \cos ^{2} x \\
y_{2}^{\prime}(x)=\quad+13 \cdot y_{3}(x)+(8 \cdot x+4) \cdot e^{2 \cdot x} \cdot \sin ^{3} x . \\
y_{3}^{\prime}(x)=-y_{1}(x) \quad+5 \cdot y_{3}(x)+(2 \cdot x+4) \cdot e^{x} \cdot \sin ^{2} x
\end{array}\right.
$$




$$
\begin{aligned}
& \left\{\begin{array}{l}
y_{1}^{\prime}(x)=\quad-y_{2}(x)+17 \cdot y_{3}(x)+e^{x}+e^{x} \cdot \cos (2 \cdot x) \\
y_{2}^{\prime}(x)=\quad+13 \cdot y_{3}(x)+(6 \cdot x+3) \cdot e^{2 \cdot x} \cdot \sin x-(2 \cdot x+1) \cdot e^{2 \cdot x} \cdot \sin (3 \cdot x) \\
y_{3}^{\prime}(x)=-y_{1}(x) \quad+5 \cdot y_{3}(x)+(x+2) \cdot e^{x}-(x+2) \cdot e^{x} \cdot \cos (2 \cdot x)
\end{array}\right. \\
& \begin{cases}y_{1}^{\prime}(x)= & -y_{2}(x)+17 \cdot y_{3}(x) \\
y_{2}^{\prime}(x)= & +13 \cdot y_{3}(x) \\
y_{3}^{\prime}(x)=-y_{1}(x) \quad+5 \cdot y_{3}(x)\end{cases}
\end{aligned}
$$

We will first determine the solutions of the characteristic equation attached to the homogeneous system (15):

$$
\left|\begin{array}{rrc}
-\lambda & -1 & 17 \\
0 & -\lambda & 13 \\
-1 & 0 & 5-\lambda
\end{array}\right|=\lambda^{2} \cdot(5-\lambda)+13-17 \cdot \lambda=0 \text {. }
$$

The solutions of this equation are $\lambda_{1}=1$ and $\lambda_{2}, \lambda_{3}=2 \pm 3 \cdot i$.

Given the form of free terms and the root of the characteristic equation (16), we will try to find a particular solution like

$$
\left\{\begin{aligned}
y_{1 p}(x) & =\left(A_{1} \cdot x+B_{1}\right) \cdot e^{x}+\left(C_{1} \cdot x+D_{1}\right) \cdot x \cdot e^{x}+\left(E_{1} \cdot x+F_{1}\right) \cdot e^{x} \cdot \cos (2 \cdot x)+ \\
& +\left(G_{1} \cdot x+H_{1}\right) \cdot e^{x} \cdot \sin (2 \cdot x)+\left(I_{1} \cdot x+J_{1}\right) \cdot e^{2 \cdot x} \cdot \cos x+\left(K_{1} \cdot x+L_{1}\right) \cdot e^{2 \cdot x} \cdot \sin x+ \\
& +\left(M_{1} \cdot x+N_{1}\right) \cdot e^{2 \cdot x} \cdot \cos (3 \cdot x)+\left(O_{1} \cdot x+P_{1}\right) \cdot e^{2 \cdot x} \cdot \sin (3 \cdot x)+ \\
& +\left(R_{1} \cdot x+S_{1}\right) \cdot x \cdot e^{2 \cdot x} \cdot \cos (3 \cdot x)+\left(T_{1} \cdot x+U_{1}\right) \cdot x \cdot e^{2 \cdot x} \cdot \sin (3 \cdot x) \\
y_{2 p}(x) & =\left(A_{2} \cdot x+B_{2}\right) \cdot e^{x}+\left(C_{2} \cdot x+D_{2}\right) \cdot x \cdot e^{x}+\left(E_{2} \cdot x+F_{2}\right) \cdot e^{x} \cdot \cos (2 \cdot x)+ \\
& +\left(G_{2} \cdot x+H_{2}\right) \cdot e^{x} \cdot \sin (2 \cdot x)+\left(I_{2} \cdot x+J_{2}\right) \cdot e^{2 \cdot x} \cdot \cos x+\left(K_{2} \cdot x+L_{2}\right) \cdot e^{2 \cdot x} \cdot \sin x+ \\
& +\left(M_{2} \cdot x+N_{2}\right) \cdot e^{2 \cdot x} \cdot \cos (3 \cdot x)+\left(O_{2} \cdot x+P_{2}\right) \cdot e^{2 \cdot x} \cdot \sin (3 \cdot x)+ \\
& +\left(R_{2} \cdot x+S_{2}\right) \cdot x \cdot e^{2 \cdot x} \cdot \cos (3 \cdot x)+\left(T_{2} \cdot x+U_{2}\right) \cdot x \cdot e^{2 \cdot x} \cdot \sin (3 \cdot x) \\
y_{3 p}(x) & =\left(A_{3} \cdot x+B_{3}\right) \cdot e^{x}+\left(C_{3} \cdot x+D_{3}\right) \cdot x \cdot e^{x}+\left(E_{3} \cdot x+F_{3}\right) \cdot e^{x} \cdot \cos (2 \cdot x)+ \\
& +\left(G_{3} \cdot x+H_{3}\right) \cdot e^{x} \cdot \sin (2 \cdot x)+\left(I_{3} \cdot x+J_{3}\right) \cdot e^{2 \cdot x} \cdot \cos x+\left(K_{3} \cdot x+L_{3}\right) \cdot e^{2 \cdot x} \cdot \sin x+ \\
& +\left(M_{3} \cdot x+N_{3}\right) \cdot e^{2 \cdot x} \cdot \cos (3 \cdot x)+\left(O_{3} \cdot x+P_{3}\right) \cdot e^{2 \cdot x} \cdot \sin (3 \cdot x)+ \\
& +\left(R_{3} \cdot x+S_{3}\right) \cdot x \cdot e^{2 \cdot x} \cdot \cos (3 \cdot x)+\left(T_{3} \cdot x+U_{3}\right) \cdot x \cdot e^{2 \cdot x} \cdot \sin (3 \cdot x)
\end{aligned}\right.
$$


The derivatives of the components of the particular solution created previously are:

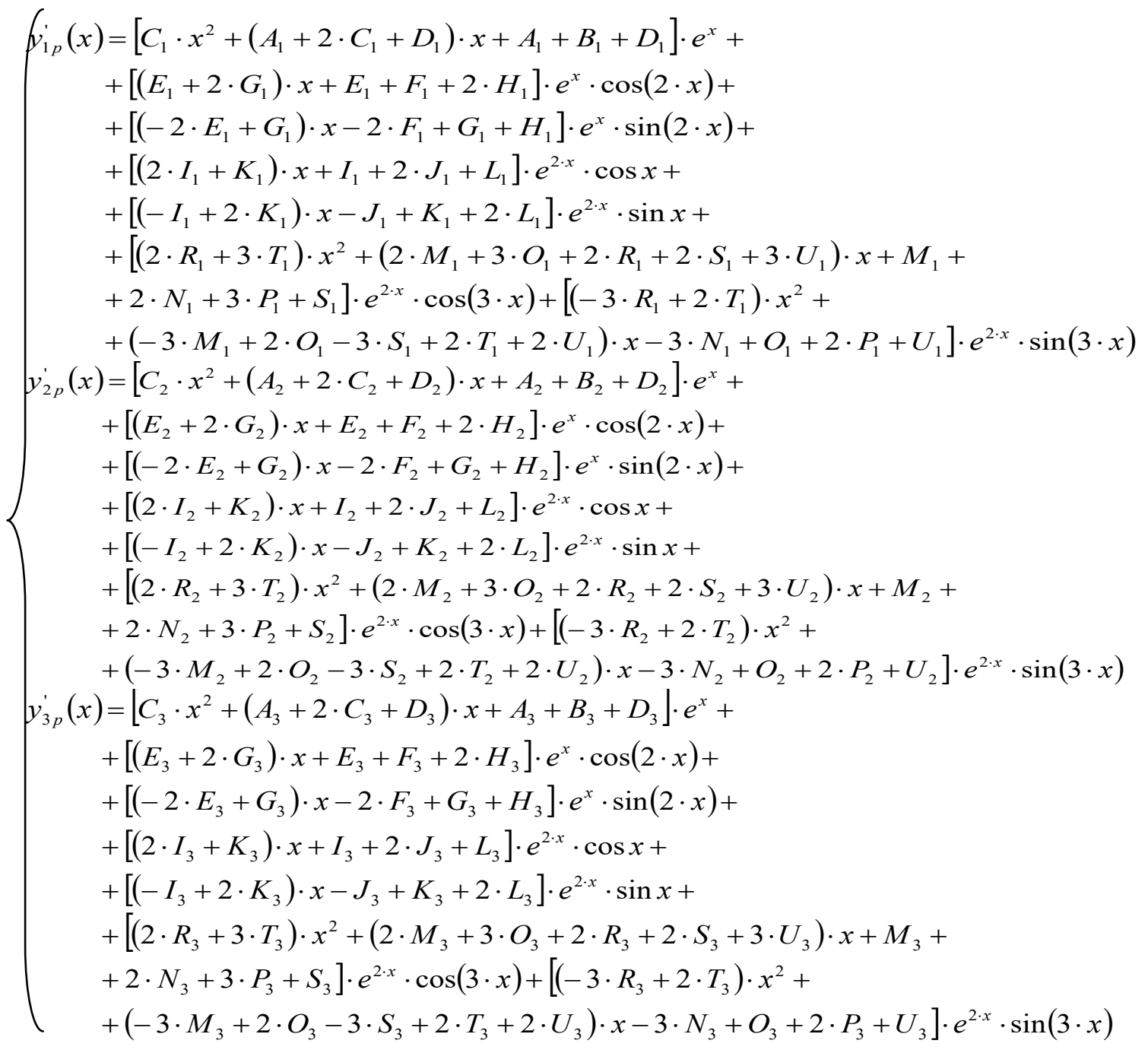


By introducing the functions $y_{1 p}, y_{2 p}$ and $y_{3 p}$, as well as their derivatives, in the system (14) we obtain, by identifying the coefficients, the following system of linear equations:

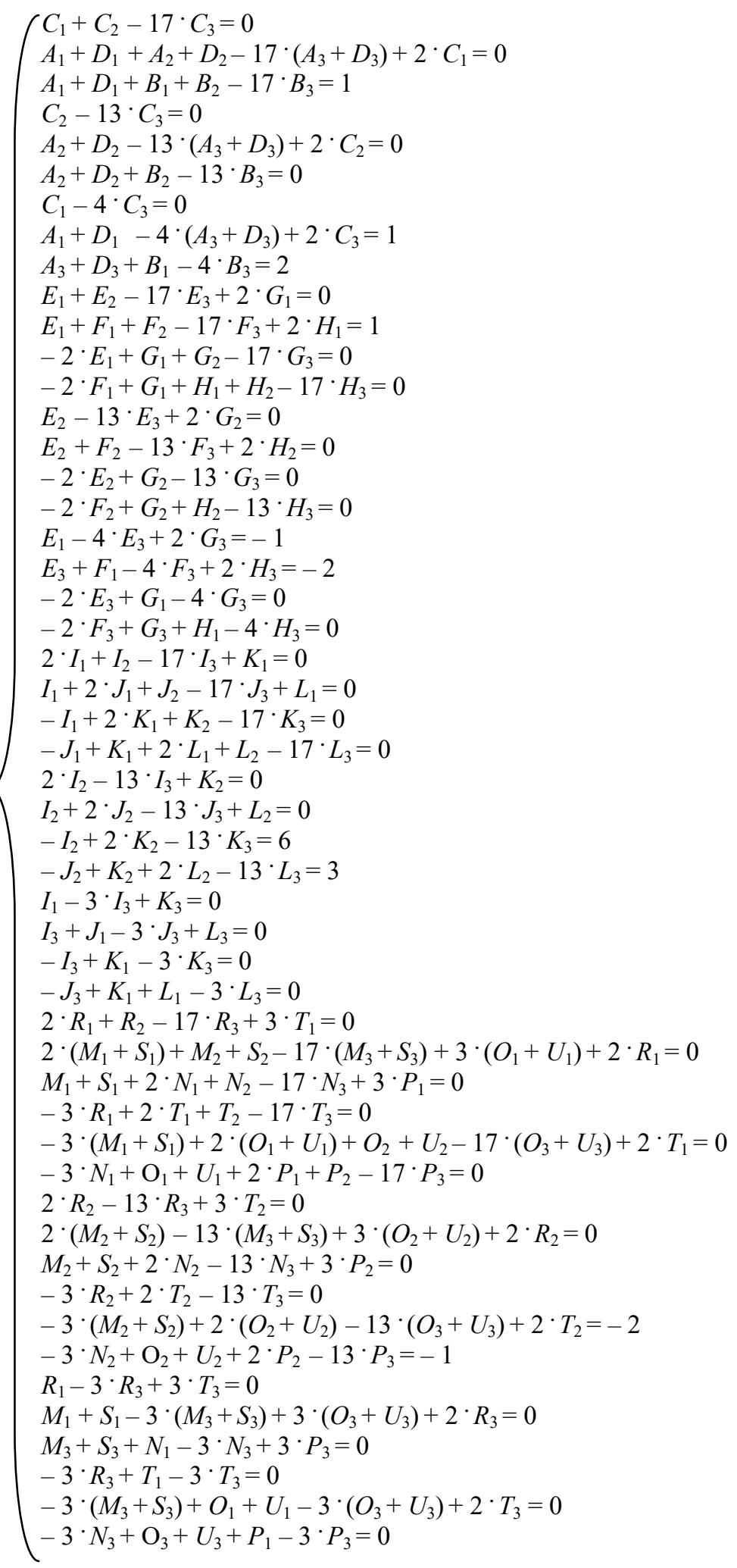




\section{The solution of the above system obtained with the Wolfram application is:}

$\ln [1]=m=\{\{\theta, \theta, \theta, \theta, \theta, \theta, 1,1,-17\},\{1,1,-17, \theta, \theta, \theta, 2, \theta, \theta\},\{1, \theta, \theta, 1,1,-17, \theta, \theta, \theta\},\{\theta, \theta, \theta, \theta, \theta, \theta, \theta, 1$,

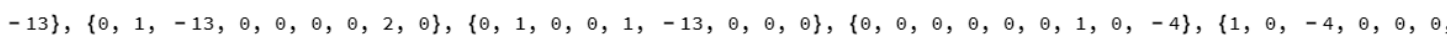
$\Theta, \Theta, 2\},\{\theta, \theta, 1,1, \theta,-4, \theta, \theta, \theta\}\}$

$\mathrm{v}=$ LinearSolve $[\mathrm{m},\{\theta, \theta, 1, \theta, \theta, \theta, \theta, 1,2\}]$

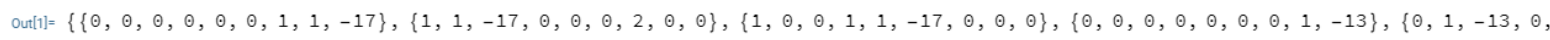

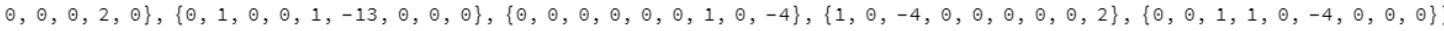

Out $[2]=\left\{\frac{109}{50}, \frac{143}{50}, \frac{8}{25}, \frac{42}{25},-\frac{143}{50}, \odot, \frac{1}{5}, \frac{13}{20}, \frac{1}{20}\right\}$

$\ln [1]=m=\{\{1,1,-17, \theta, \theta, \theta, 2, \theta, \theta, \theta, \theta, \theta\},\{1, \theta, \theta, 1,1,-17, \theta, \theta, \theta, 2, \theta, \theta\},\{-2, \theta, \theta, \theta, \theta, \theta, 1,1,-17, \theta, \theta, \theta\},\{\theta, \theta$, $\theta,-2, \theta, \theta, 1, \theta, \theta, 1,1,-17\},\{\theta, 1,-13, \theta, \theta, \theta, \theta, 2, \theta, \theta, \theta, \theta\},\{\theta, 1, \theta, \theta, 1,-13, \theta, \theta, \theta, \theta, 2, \theta\},\{\theta,-2, \theta, \theta$, $\Theta, \theta, \theta, 1,-13, \theta, \theta, \theta\},\{\theta, \theta, \theta, \theta,-2, \theta, \theta, 1, \theta, \theta, 1,-13\},\{1, \theta,-4, \theta, \theta, \theta, \theta, \theta, 2, \theta, \theta, \theta\},\{\theta, \theta, 1,1, \theta,-4$, $\Theta, \theta, \theta, \theta, \theta, 2\},\{\theta, \theta,-2, \theta, \theta, \theta, 1, \theta,-4, \theta, \theta, \theta\},\{\theta, \theta, \theta, \theta, \theta,-2, \theta, \theta, 1,1, \theta,-4\}\}$

$v=$ Linearsolve $[\mathrm{m},\{\theta, 1, \theta, \theta, \theta, \theta, \theta, \theta,-1,-2, \theta, \theta\}]$

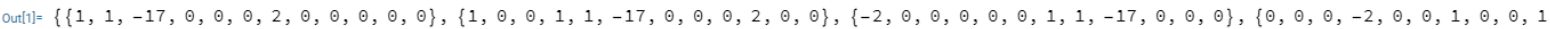

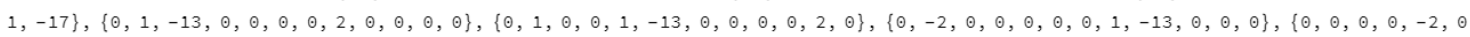

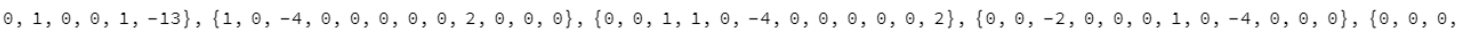
$\odot, \oplus,-2, \Theta, \odot, 1,1, \odot,-4\}\}$

Out[2] $=\left\{-\frac{55}{26},-2,-\frac{3}{26},-\frac{2187}{338},-\frac{627}{104},-\frac{935}{1352}, \frac{14}{13}, \frac{1}{4}, \frac{17}{52}, \frac{326}{169},-\frac{25}{52}, \frac{615}{676}\right\}$

$\operatorname{In}[1]=\mathrm{m}=\{\{2,1,-17, \theta, \theta, \theta, 1, \theta, \theta, \theta, \theta, \theta\},\{1, \theta, \theta, 2,1,-17, \theta, \theta, \theta, 1, \theta, \theta\},\{-1, \theta, \theta, \theta, \theta, \theta, 2,1,-17, \theta, \theta, \theta\},\{\theta, \theta$,

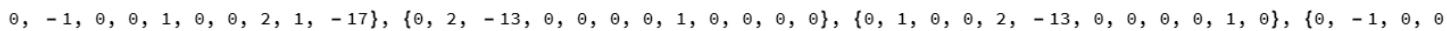

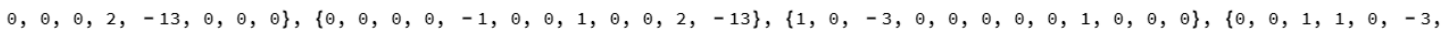

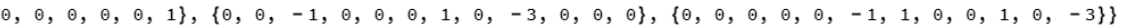

$\mathrm{v}=$ LinearSolve $[\mathrm{m},\{\theta, \theta, \theta, \theta, \theta, \theta, 6,3, \theta, \theta, \theta, \theta\}]$

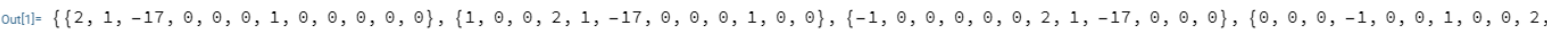

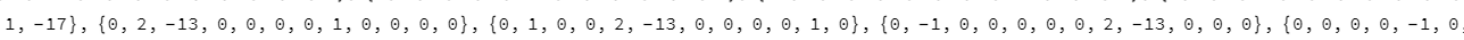

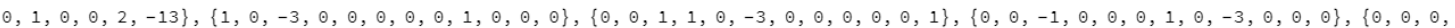
$\odot, \odot,-1,1, \odot, \odot, 1, \odot,-3\}\}$

Out $[2]=\left\{-\frac{3}{2},-\frac{33}{8},-\frac{3}{8}, \frac{21}{32}, \frac{291}{128}, \frac{9}{128}, \frac{3}{4}, \frac{27}{8}, \frac{3}{8},-\frac{57}{64}, \frac{63}{128},-\frac{9}{128}\right\}$

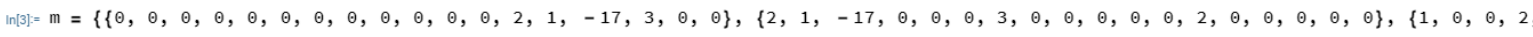

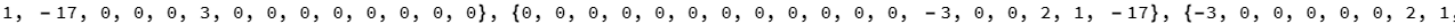

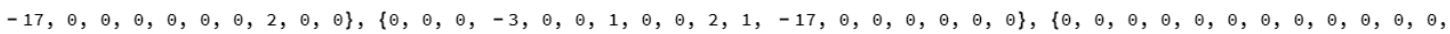

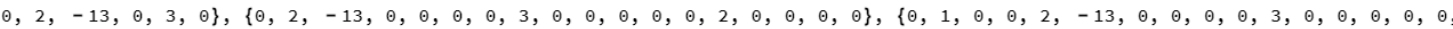
$\theta, \theta\},\{\theta, \theta, \theta, \theta, \theta, \theta, \theta, \theta, \theta, \theta, \theta, \theta, \theta,-3, \theta, \theta, 2,-13\},\{\theta,-3, \theta, \theta, \theta, \theta, \theta, 2,-13, \theta, \theta, \theta, \theta, \theta, \theta, \theta, 2, \theta\},\{\theta$,

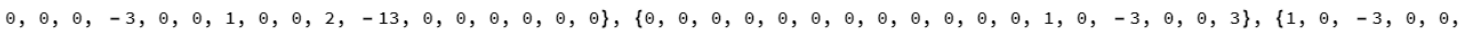

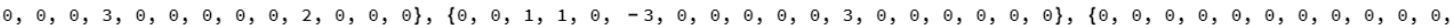

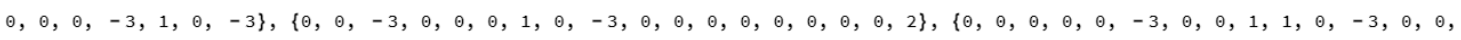
$\theta, \theta, \theta, \theta\}\}$

$v=$ LinearSolve $[\mathrm{m},\{\theta, \theta, \theta, \theta, \theta, \theta, \theta, \theta, \theta, \theta,-2,-1, \theta, \theta, \theta, \theta, \theta, \theta\}]$

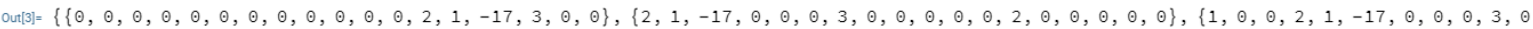

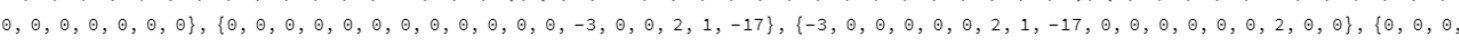

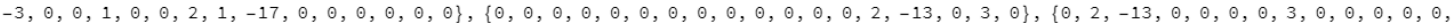

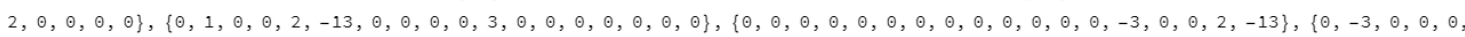

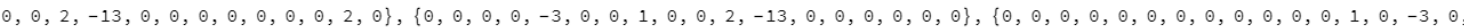

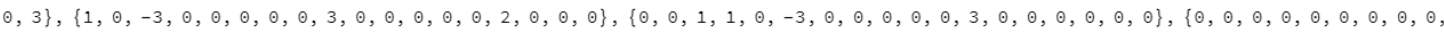

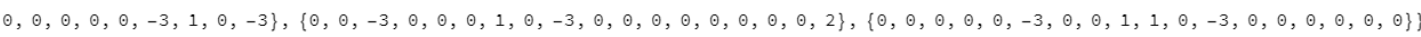

Out $[4]=\left\{\frac{11}{150}, \frac{46}{75}, \frac{3}{50}, \frac{101}{450}, \frac{209}{675}, \frac{64}{675}, \frac{23}{150},-\frac{16}{225}, \frac{11}{450}, \frac{13}{50}, \odot, \odot,-\frac{1}{10},-\frac{7}{60}, \frac{1}{60}, \frac{1}{5}, \frac{3}{20}, \frac{1}{20}\right\}$

namely, 


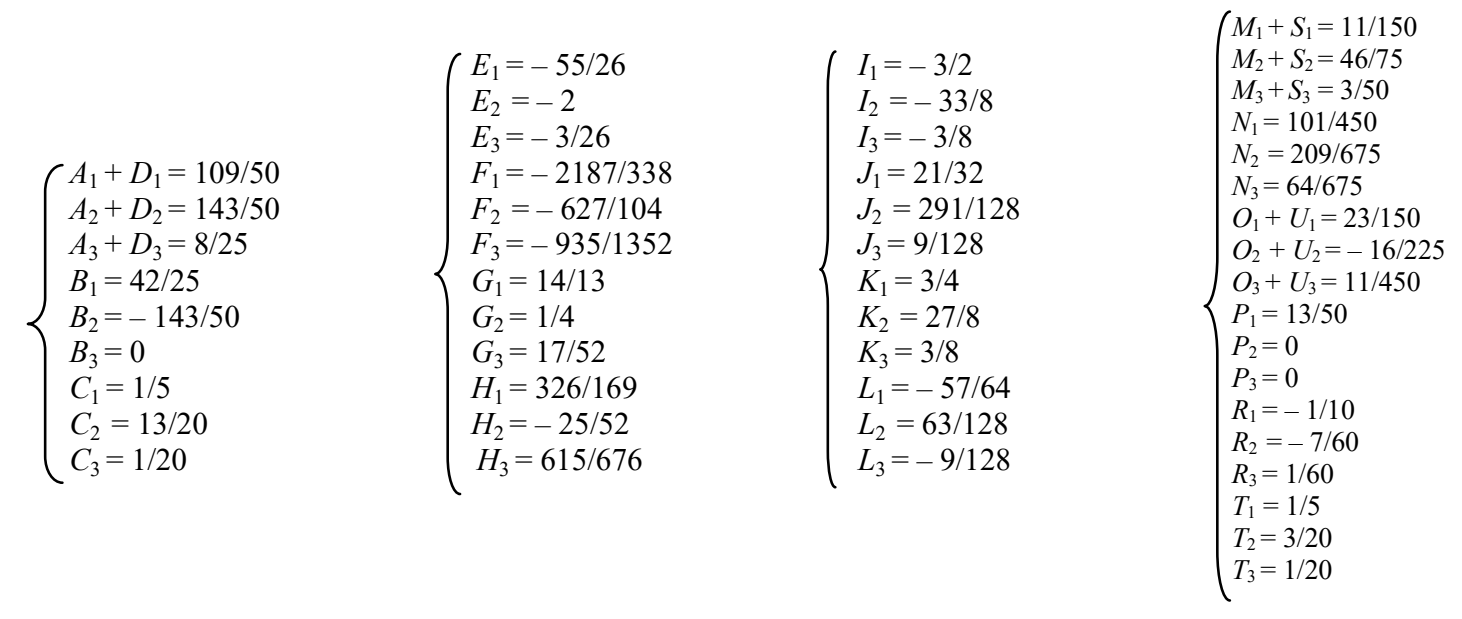

In conclusion, a particular solution of the given system (14) is

$$
\left\{\begin{aligned}
y_{1 p}(x) & =\left(\frac{1}{5} \cdot x^{2}+\frac{109}{50} \cdot x+\frac{42}{25}\right) \cdot e^{x}+\left(-\frac{55}{26} \cdot x-\frac{2187}{338}\right) \cdot e^{x} \cdot \cos (2 \cdot x)+ \\
& +\left(\frac{14}{13} \cdot x+\frac{326}{169}\right) \cdot e^{x} \cdot \sin (2 \cdot x)+\left(-\frac{3}{2} \cdot x+\frac{21}{32}\right) \cdot e^{2 \cdot x} \cdot \cos x+\left(\frac{3}{4} \cdot x-\frac{57}{64}\right) \cdot e^{2 \cdot x} \cdot \sin x+ \\
& +\left(-\frac{1}{10} \cdot x^{2}+\frac{11}{150} \cdot x+\frac{101}{450}\right) \cdot e^{2 \cdot x} \cdot \cos (3 \cdot x)+\left(\frac{1}{5} \cdot x^{2}+\frac{23}{150} \cdot x+\frac{13}{50}\right) \cdot e^{2 \cdot x} \cdot \sin (3 \cdot x) \\
& +\left(\frac{1}{4} \cdot x-\frac{25}{52}\right) \cdot e^{x} \cdot \sin (2 \cdot x)+\left(-\frac{33}{8} \cdot x+\frac{291}{128}\right) \cdot e^{2 \cdot x} \cdot \cos x+\left(\frac{27}{8} \cdot x+\frac{63}{128}\right) \cdot e^{2 \cdot x} \cdot \sin x+ \\
& +\left(-\frac{7}{60} \cdot x^{2}+\frac{46}{75} \cdot x+\frac{209}{675}\right) \cdot e^{2 \cdot x} \cdot \cos (3 \cdot x)+\left(\frac{3}{20} \cdot x^{2}-\frac{16}{225} \cdot x\right) \cdot e^{2 \cdot x} \cdot \sin (3 \cdot x) \\
y_{2 p} & =\left(\frac{13}{20} \cdot x^{2}+\frac{143}{50} \cdot x-\frac{143}{50}\right) \cdot e^{x}+\left(-2 \cdot x-\frac{627}{104}\right) \cdot e^{x} \cdot \cos (2 \cdot x)+ \\
y_{3 p}(x) & =\left(\frac{1}{20} \cdot x^{2}+\frac{8}{25} \cdot x\right) \cdot e^{x}+\left(-\frac{3}{26} \cdot x-\frac{935}{1352}\right) \cdot e^{x} \cdot \cos (2 \cdot x)+ \\
& +\left(\frac{17}{52} \cdot x+\frac{615}{676}\right) \cdot e^{x} \cdot \sin (2 \cdot x)+\left(-\frac{3}{8} \cdot x+\frac{9}{128}\right) \cdot e^{2 \cdot x} \cdot \cos x+\left(\frac{3}{8} \cdot x-\frac{9}{128}\right) \cdot e^{2 \cdot x} \cdot \sin x+ \\
& +\left(\frac{1}{60} \cdot x^{2}+\frac{3}{50} \cdot x+\frac{64}{675}\right) \cdot e^{2 \cdot x} \cdot \cos (3 \cdot x)+\left(\frac{1}{20} \cdot x^{2}+\frac{11}{450} \cdot x\right) \cdot e^{2 \cdot x} \cdot \sin (3 \cdot x)
\end{aligned}\right.
$$

After determining the particular solution, the general solution of the given system can be written.

\section{Conclusions}

This study comes to complete the range of free terms for which we can determine a particular solution in the case of a system of linear differential equations with constant coefficients. In this case, to the expression of free terms presented in [1], terms such as $P(x) \cdot e^{a \cdot x} \cdot \cos ^{n} x$ or/and $Q(x) \cdot e^{b \cdot x} \cdot \sin ^{m} x$ can be added, the particular solution being built the same as in [1], taking into account the type of functions that occur in the column of free terms. 
In the case of systems of linear differential equations with constant coefficients, too, for the construction of the particular solution, we must know which of the values generated by the terms that appear in the expression of the function $f(x)$, which brings together all functions in the column of free terms, are among the solutions of the characteristic equation and what order of multiplicity they have.

Also, in this case, determining the solution of the system of linear equations resulting from the identification of coefficients, as can be seen in the example presented is not simple, and the use of a computer application is essential.

\section{References}

[1] Vasile Căruțaşu, Determining a Particular Solution for the Systems of Linear Differential Equations with Constant Coefficients, Proceedings of the $23^{\text {rd }}$ International Conference „The Knowledge-Based Organization” of „Nicolae Bălcescu” Land Forces Academy, Sibiu, 15-17 June, 2017, pag. 30-36, ISSN 1843-6722 